\title{
Properties of $G$-martingales with finite variation and the application to $G$-Sobolev spaces
}

\author{
Yongsheng Song*
}

April 18, 2022

\begin{abstract}
As is known, a process of form $\int_{0}^{t} \eta_{s} d\langle B\rangle_{s}-\int_{0}^{t} 2 G\left(\eta_{s}\right) d s, \eta \in M_{G}^{1}(0, T)$, is a non-increasing $G$-martingale. In this paper, we shall show that a non-increasing $G$-martingale could not be form of $\int_{0}^{t} \eta_{s} d s$ or $\int_{0}^{t} \gamma_{s} d\langle B\rangle_{s}, \eta, \gamma \in M_{G}^{1}(0, T)$, which implies that the decomposition for generalized $G$-Itô processes is unique: For $\zeta \in H_{G}^{1}(0, T), \eta \in M_{G}^{1}(0, T)$ and non-increasing $G$-martingales $K, L$, if

$$
\int_{0}^{t} \zeta_{s} d B_{s}+\int_{0}^{t} \eta_{s} d s+K_{t}=L_{t}, t \in[0, T]
$$

then we have $\eta \equiv 0, \zeta \equiv 0$ and $K_{t}=L_{t}$. As an application, we give a characterization to the $G$-Sobolev spaces introduced in Peng and Song (2015).
\end{abstract}

Key words: $G$-martingales with finite variation; generalized $G$-Itô processes; unique decomposition; $G$-Sobolev spaces

MSC-classification: 60G44, 60G45, 60G48

\section{Introduction}

The notion of $G$-expectation is a type of nonlinear expectation proposed by Peng [3, 6]. It can be regarded as a nonlinear generalization of Wiener probability space $(\Omega, \mathcal{F}, P)$ where $\Omega=$ $C_{0}\left([0, \infty), \mathbb{R}^{d}\right)$ equipped with the uniform norm, $\mathcal{F}=\mathcal{B}(\Omega)$ and $P$ is a Wiener probability measure defined on $(\Omega, \mathcal{F})$. Recall that the Wiener measure is defined such that the canonical process $B_{t}(\omega):=\omega_{t}, t \geq 0$ is a continuous process with stationary and independent increments, namely $\left(B_{t}\right)_{t \geq 0}$ is a Brownian motion. $G$-expectation $\mathbb{E}$ is a sublinear expectation on the same canonical space $\Omega$, such that the same canonical process $B$ is a $G$-Brownian motion, i.e., it is a continuous process with stationary and independent increments. A crucial difference is that the quadratic variance process $\langle B\rangle$ of the $G$-Brownian motion $B$ is no longer a deterministic function of the time variable $t$. It is a process with stationary and independent increments. For the one-dimensional case, its increments are bounded by $\bar{\sigma}^{2}:=\mathbb{E}\left[B_{1}^{2}\right] \geq-\mathbb{E}\left[-B_{1}^{2}\right]=: \underline{\sigma}^{2}$,

$$
\underline{\sigma}^{2}(t-s) \leq\langle B\rangle_{t}-\langle B\rangle_{s} \leq \bar{\sigma}^{2}(t-s) \text {, for } s<t .
$$

Similar to the classical Brownian motion, the G-Brownian motion corresponds to a (fully nonlinear) PDE: For a function $\varphi \in C_{b, L i p}(\mathbb{R})$, the collection of bounded Lipstchiz functions

*Academy of Mathematics and Systems Science, CAS, Beijing, China, yssong@amss.ac.cn. Research supported by NCMIS; Key Project of NSF (No. 11231005); Key Lab of Random Complex Structures and Data Science, CAS (No. 2008DP173182). 
on $\mathbb{R}$, the function $u(t, x):=\mathbb{E}\left[\varphi\left(x+B_{t}\right)\right]$ is the (viscosity) solution to the following $G$-heat equation

$$
\begin{aligned}
\partial_{t} u-G\left(\partial_{x}^{2} u\right) & =0,(t, x) \in(0, \infty) \times \mathbb{R}, \\
u(0, x) & =\varphi(x),
\end{aligned}
$$

where $G(a)=\frac{1}{2}\left(\bar{\sigma}^{2} a^{+}-\underline{\sigma}^{2} a^{-}\right), a \in \mathbb{R}$. Moreover, for fixed $T>0$, the process $u\left(T-t, B_{t}\right)$, $t \in[0, T]$ is a martingale under $G$-expectation. By Itô's formula, one has

$$
\begin{aligned}
u\left(T-t, B_{t}\right)= & \mathbb{E}\left[\varphi\left(B_{T}\right)\right]+\int_{0}^{t} \partial_{x} u\left(T-s, B_{s}\right) d B_{s} \\
& +\frac{1}{2} \int_{0}^{t} \partial_{x}^{2} u\left(T-s, B_{s}\right) d\langle B\rangle_{s}-\int_{0}^{t} G\left(\partial_{x}^{2} u\right)\left(T-s, B_{s}\right) d s .
\end{aligned}
$$

The process $M_{t}:=\int_{0}^{t} \partial_{x} u\left(T-s, B_{s}\right) d B_{s}$ is a symmetric $G$-martingale (i.e., $M$ and $-M$ are both $G$-martingales), which shares the same properties with classical martingales in the probability space. The process $K_{t}:=\frac{1}{2} \int_{0}^{t} \partial_{x}^{2} u\left(T-s, B_{s}\right) d\langle B\rangle_{s}-\int_{0}^{t} G\left(\partial_{x}^{2} u\right)\left(T-s, B_{s}\right) d s$ is a non-increasing $G$-martingale. For the linear case $(\underline{\sigma}=\bar{\sigma})$, this term disappears. However, when $\underline{\sigma}<\bar{\sigma}, G$ martingales with finite variation are a class of nontrivial processes, which show the variance uncertainty of $G$-expectation.

For $Z \in H_{G}^{2}(0, T), \eta \in M_{G}^{2}(0, T)$, [4 showed that a process of form

$$
X_{t}=X_{0}+\int_{0}^{t} Z_{s} d B_{s}+\int_{0}^{t} \eta_{s} d\langle B\rangle_{s}-\int_{0}^{t} 2 G\left(\eta_{s}\right) d s
$$

is a $G$-martingale, and conjectured that for any $\xi \in L_{G}^{2}\left(\Omega_{T}\right)$, the martingale $\mathbb{E}_{t}[\xi]$ has the representation (1.2) . 4 proved this conjecture for cylinder random variables of form $\xi=\varphi\left(B_{t_{1}}, \cdot \cdot\right.$ $\left.\cdot, B_{t_{n}}\right)$. For the general case, Soner et al (2011) and Song (2011) proved independently the following $G$-martingale decomposition theorem:

$$
\mathbb{E}_{t}[\xi]=\mathbb{E}[\xi]+\int_{0}^{t} Z_{s} d B_{s}+\int_{0}^{t} \eta_{s} d\langle B\rangle_{s}+K_{t}
$$

where $K_{t}$ is a non-increasing $G$-martingale.

In this paper, our interest concentrates on $G$-martingales with finite variation. In the $G$ expectation space, there are three types of processes whose variation is finite.

(1) $L_{t}=\int_{0}^{t} \eta_{s} d s, \eta \in M_{G}^{p}(0, T)$;

(2) $A_{t}=\int_{0}^{t} \zeta_{s} d\langle B\rangle_{s}, \zeta \in M_{G}^{p}(0, T)$;

(3) $G$-martingales with finite variation.

It is a very important problem to distinguish these three types of processes. Song (2012) distinguished (1) and (2) completely:

$$
\int_{0}^{t} \eta_{s} d s=\int_{0}^{t} \zeta_{s} d\langle B\rangle_{s}, t \in[0, T] \Longrightarrow \eta \equiv \zeta=0 .
$$

As an immediate corollary of this result, Song (2012) proved the uniqueness of the representation for $G$-martingales with finite variation. Also, Conclusion (1.3) implies that the decomposition of $G$-Itô process is unique, which is crucial for Peng and Song (2015) to define the $G$-Sobolev space $W_{G}^{1,2 ; p}(0, T)$. 
The main job of this paper is to distinguish $G$-martingales with finite variation from the other two types of processes. For a $G$-martingale of the form $K_{t}(\varsigma)=\int_{0}^{t} \varsigma_{s} d\langle B\rangle_{s}-\int_{0}^{t} 2 G\left(\varsigma_{s}\right) d s$, if $K_{t}(\varsigma)=\int_{0}^{t} \eta_{s} d s$ (resp. $\left.\int_{0}^{t} \zeta_{s} d\langle B\rangle_{s}\right), t \in[0, T]$, then by Conclusion (1.3), we get $\varsigma \equiv \eta=0$ (resp. $\varsigma \equiv \zeta=0)$. So a $G$-martingale $K_{t}(\varsigma)$ could not be form of (1) or (2). Here we shall prove this conclusion for general $G$-martingales:

A $G$-martingale with finite variation could not be form of $\int_{0}^{t} \eta_{s} d s$ or $\int_{0}^{t} \zeta_{s} d\langle B\rangle_{s}$.

More precisely, let $K$ be a non-increasing $G$-martingale. If

$$
K_{t}=\int_{0}^{t} \eta_{s} d s\left(\operatorname{resp} . \int_{0}^{t} \zeta_{s} d\langle B\rangle_{s}\right), t \in[0, T]
$$

we conclude that $K \equiv 0$.

Based on this conclusion, we can prove that the decomposition for generalized $G$-Itô processes is unique: For $\zeta \in H_{G}^{1}(0, T), \eta \in M_{G}^{1}(0, T)$ and non-increasing $G$-martingales $K, L$, if

$$
\int_{0}^{t} \zeta_{s} d B_{s}+\int_{0}^{t} \eta_{s} d s+K_{t}=L_{t}, t \in[0, T]
$$

then we have $\eta \equiv 0, \zeta \equiv 0$ and $K_{t}=L_{t}$. This turns out to be a very strong result. Many important conclusions in the context of $G$-expectation theory, including Conclusion (1.3), can be considered as its immediate corollaries (see Remark 3.12 for details). The main results of this paper are Theorem 3.6 and Theorem 3.10.

Peng and Song (2015) introduced the notion of $G$-Sobolev spaces. In the $G$-Sobolev space $W_{\mathcal{A}_{G}}^{\frac{1}{2}, 1 ; p}(0, T)$ the authors defined solutions to the following path dependent PDEs:

$$
\begin{aligned}
\mathcal{D}_{t} u+G\left(\mathcal{D}_{x}^{2} u\right)+f\left(t, u, \mathcal{D}_{x} u\right) & =0, \quad t \in[0, T), \\
u_{T} & =\xi .
\end{aligned}
$$

This $W_{\mathcal{A}_{G}}^{\frac{1}{2}, 1 ; p}$-solution corresponds to the solution of the backward SDEs driven by $G$-Brownian motion considered in $\mathrm{Hu}$ et al (2014).

In this paper, as an application of the main results, we shall give a characterization of the $G$-Sobolev space $W_{\mathcal{A}_{G}}^{\frac{1}{2}, 1 ; p}(0, T)$. The main idea is, just like the liner case, to integrate $\mathcal{A}_{G} u=$ $\mathcal{D}_{t} u+G\left(\mathcal{D}_{x}^{2} u\right)$ as one operator, which reduces the regularity requirement for the solutions. To well define the derivative $\mathcal{A}_{G} u$ for $u \in W_{\mathcal{A}_{G}}^{\frac{1}{2}, 1 ; p}(0, T)$, the uniqueness of the decomposition for generalized $G$-Itô processes plays a crucial role.

The rest of the paper is organized as follows. In Section 2, we present some basic notions and definitions on the $G$-expectation theory. We shall prove the main results in Section 3. As an application of the uniqueness of the decomposition for generalized $G$-Itô processes, we shall refine the definition of the $G$-Sobolev space $W_{\mathcal{A}_{G}}^{\frac{1}{2}, 1 ; p}$ in Section 4 . In Section 5, as an appendix, we present the wellposedness result of $G$-BSDEs obtained in [2].

\section{Some definitions and notations about $G$-expectation}

We review some basic notions and definitions on the $G$-expectation theory. The readers may refer to [3], 4], [5], 6] for more details.

Let $\Omega_{T}=C_{0}\left([0, T] ; \mathbb{R}^{d}\right)$ be the space of all $\mathbb{R}^{d}$-valued continuous paths $\omega=(\omega(t))_{t \in[0, T]}$ with $\omega(0)=0$ and let $B_{t}(\omega)=\omega(t)$ be the canonical process. 
Let us recall the definitions of $G$-Brownian motion and its corresponding $G$-expectation introduced in [4]. For simplicity, here we only consider the one-dimensional case.

Set

$$
L_{i p}\left(\Omega_{T}\right):=\left\{\varphi\left(\omega\left(t_{1}\right), \cdots, \omega\left(t_{n}\right)\right): t_{1}, \cdots, t_{n} \in[0, T], \varphi \in C_{b, L i p}\left(\mathbb{R}^{n}\right), n \in \mathbb{N}\right\},
$$

where $C_{b, \text { Lip }}\left(\mathbb{R}^{n}\right)$ is the collection of bounded Lipschitz functions on $\mathbb{R}^{n}$.

We are given a function $G: \mathbb{R} \mapsto \mathbb{R}$, for $0 \leq \underline{\sigma}^{2} \leq \bar{\sigma}^{2}$, by

$$
G(a):=\frac{1}{2}\left(\bar{\sigma}^{2} a^{+}-\underline{\sigma}^{2} a^{-}\right) .
$$

For each $\xi \in L_{i p}\left(\Omega_{T}\right)$ of the form

$$
\xi(\omega)=\varphi\left(\omega\left(t_{1}\right), \omega\left(t_{2}\right), \cdots, \omega\left(t_{n}\right)\right), \quad 0=t_{0}<t_{1}<\cdots<t_{n}=T,
$$

we define the following conditional $G$-expectation

$$
\mathbb{E}_{t}[\xi]:=u_{k}\left(t, \omega(t) ; \omega\left(t_{1}\right), \cdots, \omega\left(t_{k-1}\right)\right)
$$

for each $t \in\left[t_{k-1}, t_{k}\right), k=1, \cdots, n$. Here, for each $k=1, \cdots, n, u_{k}=u_{k}\left(t, x ; x_{1}, \cdots, x_{k-1}\right)$ is a function of $(t, x)$ parameterized by $\left(x_{1}, \cdots, x_{k-1}\right) \in \mathbb{R}^{k-1}$, which is the solution of the following PDE (G-heat equation) defined on $\left[t_{k-1}, t_{k}\right) \times \mathbb{R}$ :

$$
\partial_{t} u_{k}+G\left(\partial_{x}^{2} u_{k}\right)=0
$$

with terminal conditions

$$
u_{k}\left(t_{k}, x ; x_{1}, \cdots, x_{k-1}\right)=u_{k+1}\left(t_{k}, x ; x_{1}, \cdots x_{k-1}, x\right), \text { for } k<n
$$

and $u_{n}\left(t_{n}, x ; x_{1}, \cdots, x_{n-1}\right)=\varphi\left(x_{1}, \cdots x_{n-1}, x\right)$.

The $G$-expectation of $\xi$ is defined by $\mathbb{E}[\xi]=\mathbb{E}_{0}[\xi]$. From this construction we obtain a natural norm $\|\xi\|_{L_{G}^{p}}:=\mathbb{E}\left[|\xi|^{p}\right]^{1 / p}, p \geq 1$. The completion of $L_{i p}\left(\Omega_{T}\right)$ under $\|\cdot\|_{L_{G}^{p}}$ is a Banach space, denoted by $L_{G}^{p}\left(\Omega_{T}\right)$. The canonical process $B_{t}(\omega):=\omega(t), t \geq 0$, is called a $G$-Brownian motion in this sublinear expectation space $\left(\Omega_{T}, L_{G}^{1}\left(\Omega_{T}\right), \mathbb{E}\right)$.

Remark 2.1 For $\varepsilon \in\left[0, \frac{\bar{\sigma}^{2}-\underline{\sigma}^{2}}{2}\right]$, set $G_{\varepsilon}(a)=G(a)-\frac{\varepsilon}{2}|a|$. Sometimes, we denote by $\mathbb{E}_{G_{\varepsilon}}$ the $G$-expectation corresponds to the function $G_{\varepsilon}$.

Definition 2.2 A process $\left\{M_{t}\right\}$ with values in $L_{G}^{1}\left(\Omega_{T}\right)$ is called a $G$-martingale if $\mathbb{E}_{s}\left(M_{t}\right)=M_{s}$ for any $s \leq t$. If $\left\{M_{t}\right\}$ and $\left\{-M_{t}\right\}$ are both $G$-martingales, we call $\left\{M_{t}\right\}$ a symmetric $G$ martingale.

Theorem 2.3 ([1]) There exists a tight subset $\mathcal{P} \subset \mathcal{M}_{1}\left(\Omega_{T}\right)$, the set of probability measures on $\left(\Omega_{T}, \mathcal{B}\left(\Omega_{T}\right)\right)$, such that

$$
\mathbb{E}[\xi]=\sup _{Q \in \mathcal{P}} E_{Q}[\xi] \text { for all } \xi \in L_{i p}\left(\Omega_{T}\right) .
$$

$\mathcal{P}$ is called a set that represents $\mathbb{E}$.

Remark 2.4 Let $W_{t}$ be a one-dimensional standard Brownian motion in the probability space $(\Omega, \mathcal{F}, P)$ and let $\mathbb{F}:=\left(\mathcal{F}_{t}\right)_{t \geq 0}$ be the augmented filtration generated by $\left(W_{t}\right)_{t \geq 0}$. Denote by $\mathcal{L}_{\mathbb{F}}^{G}$ the set of $\mathbb{F}$-adapted measurable processes with values in $[\underline{\sigma}, \bar{\sigma}]$. [1] showed that

$$
\mathcal{P}_{G}:=\left\{P_{h} \mid P_{h}:=P \circ\left(\int_{0} h_{s} d W_{s}\right)^{-1}, h \in \mathcal{L}_{\mathbb{F}}^{G}\right\}
$$

is a set that represents $\mathbb{E}$. 
Definition 2.5 A function $\eta(t, \omega):[0, T] \times \Omega_{T} \rightarrow \mathbb{R}$ is called a step process if there exists a time partition $\left\{t_{i}\right\}_{i=0}^{n}$ with $0=t_{0}<t_{1}<\cdots<t_{n}=T$, such that for each $k=0,1, \cdots, n-1$ and $t \in\left(t_{k}, t_{k+1}\right]$

$$
\eta(t, \omega)=\xi_{t_{k}} \in L_{i p}\left(\Omega_{t_{k}}\right)
$$

We denote by $M^{0}(0, T)$ the collection of all step processes.

For each $p \geq 1$, we denote by $M_{G}^{p}(0, T)$ the completion of the space $M^{0}(0, T)$ under the norm

$$
\|\eta\|_{M_{G}^{p}}:=\left\{\mathbb{E}\left[\int_{0}^{T}\left|\eta_{t}\right|^{p} d t\right]\right\}^{1 / p},
$$

and by $H_{G}^{p}(0, T)$ the completion of the space $M^{0}(0, T)$ under the norm

$$
\|\eta\|_{H_{G}^{p}}:=\left[\mathbb{E}\left[\left\{\int_{0}^{T}\left|\eta_{t}\right|^{2} d t\right]\right\}^{p / 2}\right]^{1 / p} .
$$

Theorem 2.6 ([8], [9]) For $\xi \in L_{G}^{\beta}\left(\Omega_{T}\right)$ with some $\beta>1, X_{t}=\mathbb{E}_{t}(\xi), t \in[0, T]$ has the following decomposition:

$$
X_{t}=\mathbb{E}[\xi]+\int_{0}^{t} Z_{s} d B_{s}+K_{t}, q . s .,
$$

where $\left\{Z_{t}\right\} \in H_{G}^{1}(0, T)$ and $\left\{K_{t}\right\}$ is a continuous non-increasing G-martingale. Furthermore, the above decomposition is unique and $\left\{Z_{t}\right\} \in H_{G}^{\alpha}(0, T), K_{T} \in L_{G}^{\alpha}\left(\Omega_{T}\right)$ for any $1 \leq \alpha<\beta$.

\section{Main results}

In the sequel, we shall only consider the one-dimensional $G$-expectation space which is nondegenerate and really nonlinear, i.e., $\bar{\sigma}>\underline{\sigma}>0$.

Let $W$ be a standard Brownian motion in the probability space $(\Omega, \mathcal{F}, P)$ and assume that $\mathbb{F}=\left(\mathcal{F}_{t}\right)$ is the augmented filtration generated by $W$.

An $\mathbb{F}$-adapted measurable process $h$ is called an (m-steps) self-dependent process if it has the following form:

$$
h_{t}=\sum_{i=0}^{m-1} \xi_{i} 1_{] \frac{i}{m}, \frac{i+1}{m}\right]}(t)
$$

where $\xi_{i}=\varphi_{i}\left(\int_{\frac{i-1}{m}}^{\frac{i}{m}} h_{s} d W_{s}, \cdots, \int_{0}^{\frac{1}{m}} h_{s} d W_{s}\right), \varphi_{i} \in C_{b, L i p}\left(R^{i}\right)$. Clearly, an $m$-steps self-dependent process $h$ can also be considered as a $2^{n} m$-steps self-dependent process for any $n \geq 0$.

Lemma 3.1 (Lemma 4.2 in [11]) The collection of self-dependent processes bounded by two positive constants $c, C\left(c \leq\left|h_{s}\right| \leq C\right)$ is dense in the collection of $\mathbb{F}$-adapted measurable processes bounded by the same constants $c, C$ under the norm

$$
\|h\|_{2}=\left[E\left(\int_{0}^{1}\left|h_{s}\right|^{2} d s\right)\right]^{1 / 2} .
$$

Let $B_{t}(\omega)=\omega_{t}$ be the canonical process on the space $\Omega_{T}$. For an $\mathbb{F}$-adapted measurable process $h$, set $P_{h}=P \circ\left(\int_{0}^{\cdot} h_{s} d W_{s}\right)^{-1}$, a probability on $\left(\Omega_{T}, \mathcal{B}\left(\Omega_{T}\right)\right)$. For a process $\left\{X_{t}\right\}$, we denote by $X_{[0, T]}^{m}$ the vector $\left(X_{T}-X_{\frac{(m-1) T}{m}}, \cdots, X_{\frac{T}{m}}-X_{0}\right)$. 
Lemma 3.2 Let $h$ be an m-steps self-dependent process of form (3.1). We call a bounded $\mathbb{F}$ adapted measurable process $\tilde{h}$ an m-perturbation of $h$ if the following property holds:

$$
\int_{\frac{i}{m}}^{\frac{i+1}{m}}\left|\tilde{h}_{s}\right|^{2} d s=\frac{1}{m}\left|\tilde{\xi}_{i}\right|^{2}:=\frac{1}{m}\left|\varphi_{i}\left(\int_{\frac{i-1}{m}}^{\frac{i}{m}} \tilde{h}_{s} d W_{s}, \cdots, \int_{0}^{\frac{1}{m}} \tilde{h}_{s} d W_{s}\right)\right|^{2}, \text { P-a.s. }
$$

Then for any random variable of the form $X=\psi\left(B_{[0,1]}^{m}\right)$ with $\psi$ a bounded Lipschiz continuous function, we have

$$
E_{P_{h}}[X]=E_{P_{\tilde{h}}}[X] .
$$

Proof. Set $\psi_{1}\left(x_{m-1}, \cdots, x_{1}\right):=E\left[\psi\left(\varphi_{m-1}\left(x_{m-1}, \cdots, x_{1}\right)\left(W_{1}-W_{\frac{m-1}{m}}\right), x_{m-1}, \cdots, x_{1}\right)\right]$. On the one hand, we have

$$
\begin{aligned}
E_{P_{h}}[X] & =E\left[\psi\left(\int_{\frac{m-1}{m}}^{1} h_{s} d W_{s}, \cdots, \int_{0}^{\frac{1}{m}} h_{s} d W_{s}\right)\right] \\
& =E\left[E\left[\psi\left(\int_{\frac{m-1}{m}}^{1} h_{s} d W_{s}, \cdots, \int_{0}^{\frac{1}{m}} h_{s} d W_{s}\right) \mid \mathcal{F}_{\frac{m-1}{m}}\right]\right] \\
& =E\left[\psi_{1}\left(\int_{\frac{m-2}{m}}^{\frac{m-1}{m}} h_{s} d W_{s}, \cdots, \int_{0}^{\frac{1}{m}} h_{s} d W_{s}\right)\right] .
\end{aligned}
$$

On the other hand, letting $P_{\frac{m-1}{m}}^{\omega}$ be the regular conditional probability of $P\left(\cdot \mid \mathcal{F}_{\frac{m-1}{m}}\right), \int_{\frac{m-1}{m}}^{1} \tilde{h}_{s} d W_{s}$ is normally distributed under $P_{\frac{m-1}{m}}^{\omega}$ with mean 0 and variance $\frac{1}{m}\left|\tilde{\xi}_{m-1}\right|^{2}(\omega)$ since $\int_{\frac{i}{m}}^{\frac{i+1}{m}}\left|\tilde{h}_{s}\right|^{2} d s=$ $\frac{1}{m}\left|\tilde{\xi}_{i}\right|^{2}$. So

$$
\begin{aligned}
E_{P_{\tilde{h}}}[X] & =E\left[\psi\left(\int_{\frac{m-1}{m}}^{1} \tilde{h}_{s} d W_{s}, \cdots, \int_{0}^{\frac{1}{m}} \tilde{h}_{s} d W_{s}\right)\right] \\
& =E\left[E\left[\psi\left(\int_{\frac{m-1}{m}}^{1} \tilde{h}_{s} d W_{s}, \cdots, \int_{0}^{\frac{1}{m}} \tilde{h}_{s} d W_{s}\right) \mid \mathcal{F}_{\frac{m-1}{m}}\right]\right] \\
& =E\left[\psi_{1}\left(\int_{\frac{m-2}{m}}^{\frac{m-1}{m}} \tilde{h}_{s} d W_{s}, \cdots, \int_{0}^{\frac{1}{m}} \tilde{h}_{s} d W_{s}\right)\right] .
\end{aligned}
$$

Repeating the above arguments for $m-1$ times, finally we can find a bounded Lipschiz continuous function $\psi_{m-1}$ such that

$$
E_{P_{h}}[X]=E_{P}\left[\psi_{m-1}\left(\int_{0}^{\frac{1}{m}} h_{s} d W_{s}\right)\right], E_{P_{\tilde{h}}}[X]=E_{P}\left[\psi_{m-1}\left(\int_{0}^{\frac{1}{m}} \tilde{h}_{s} d W_{s}\right)\right] .
$$

Since $\int_{0}^{t}\left|\tilde{h}_{s}\right|^{2} d s=\frac{1}{m} \tilde{\xi}_{0}^{2}=\frac{1}{m} \xi_{0}^{2}, \int_{0}^{\frac{1}{m}} h_{s} d W_{s}$ and $\int_{0}^{\frac{1}{m}} \tilde{h}_{s} d W_{s}$ are both normally distributed with mean 0 and variance $\frac{1}{m}\left|\xi_{0}\right|^{2}$. Hence, we have $E_{P_{h}}[X]=E_{P_{\tilde{h}}}[X]$.

Theorem 3.3 Let $h$ be an m-steps self-dependent process. For $n \geq 1$, let $h^{n}$ be a $2^{n} m$ perturbation of $h$. Assuming that $\left(h^{n}\right)_{n \geq 1}$ are uniformly bounded, we have

$$
P_{h^{n}} \stackrel{w}{\rightarrow} P_{h}
$$


Proof. For any $k \geq 1$ and any function $\psi \in C_{b, \text { Lip }}\left(R^{2^{k} m}\right)$, by Lemma 3.2, we have, for $n \geq k$,

$$
E_{P_{h^{n}}}\left[\psi\left(B_{[0,1]}^{2^{k} m}\right)\right]=E_{P_{h}}\left[\psi\left(B_{[0,1]}^{2^{k} m}\right)\right] .
$$

In other words, we have

$$
\lim _{n \rightarrow \infty} E_{P_{h^{n}}}\left[\psi\left(B_{[0,1]}^{2^{k} m}\right)\right]=E_{P_{h}}\left[\psi\left(B_{[0,1]}^{2^{k} m}\right)\right]
$$

for any $k \geq 1$ and any function $\psi \in C_{b, \text { Lip }}\left(R^{2^{k} m}\right)$.

Since $\left(h^{n}\right)_{n \geq 1}$ are uniformly bounded, we know that $\left(P_{h^{n}}\right)_{n \geq 1}$ are tight. Combing the above arguments, we conclude that

$$
P_{h^{n}} \stackrel{w}{\rightarrow} P_{h}
$$

Lemma 3.4 Let $K$ be a non-increasing $G$-martingale. Fix an $\mathbb{F}$-adapted measurable process $h$ with $\underline{\sigma} \leq|h| \leq \bar{\sigma}$. Then for any $s<t$ and any $\varepsilon>0$ there exists an $\mathbb{F}$-adapted measurable process $\tilde{h}$ with $\underline{\sigma} \leq|\tilde{h}| \leq \bar{\sigma}$ and $\tilde{h}_{r} 1_{[0, s]}(r)=h_{r} 1_{[0, s]}(r)$ such that $E_{P_{\tilde{h}}}\left[-\left(K_{t}-K_{s}\right)\right]<\varepsilon$.

Proof. Fix $s<t, \varepsilon>0$ and an $\mathbb{F}$-adapted measurable process $h$ with $\underline{\sigma} \leq|h| \leq \bar{\sigma}$. By Theorem 5.4 in [10], for the non-increasing $G$-martingale $K_{t}$, there exist $\zeta \in M^{0}(0, T)$ such that

$$
\mathbb{E}\left[\sup _{r \in[0,1]}\left|K_{r}-K_{r}(\zeta)\right|\right]<\frac{\varepsilon}{2},
$$

where $K_{r}(\zeta)=\int_{0}^{r} \zeta_{u} d\langle B\rangle_{u}-\int_{0}^{r} 2 G\left(\zeta_{u}\right) d u$. We assume that $\zeta$ is of the following form:

$$
\zeta_{u}=\sum_{i=0}^{m-1} a_{t_{i}} 1_{] t_{i}, t_{i+1}\right]}(u),
$$

where $a_{t_{i}}=\phi_{i}\left(B_{t_{i}}-B_{t_{i-1}}, \cdots, B_{t_{1}}\right)$ with $\phi_{i} \in C_{b, L i p}\left(R^{i}\right)$. Without loss of generality, we assume $s=t_{i}$ and $t=t_{i+1}$. Set $\tilde{a}_{t_{i}}=\phi_{i}\left(\int_{t_{i-1}}^{t_{i}} h_{u} d W_{u}, \cdots, \int_{0}^{t_{1}} h_{u} d W_{u}\right)$ and

$$
\operatorname{sign}_{\underline{\sigma}, \bar{\sigma}}\left(\tilde{a}_{t_{i}}\right)= \begin{cases}\bar{\sigma} & \text { if } \tilde{a}_{t_{i}} \geq 0 \\ \underline{\sigma} & \text { if } \tilde{a}_{t_{i}}<0 .\end{cases}
$$

Let $\tilde{h}_{r}=h_{r}$ for $s \in\left[0, t_{i}\right]$ and let $\tilde{h}_{r}=\operatorname{sign}_{\underline{\sigma}, \bar{\sigma}}\left(\tilde{a}_{t_{i}}\right)$ for $\left.\left.r \in\right] t_{i}, t_{i+1}\right]$. Then

$$
\begin{aligned}
E_{P_{\tilde{h}}}\left[K_{t}(\zeta)-K_{s}(\zeta)\right] & =E_{P_{\tilde{h}}}\left[a_{t_{i}}\left(\langle B\rangle_{t_{i+1}}-\langle B\rangle_{t_{i}}\right)-2 G\left(a_{t_{i}}\right)\left(t_{i+1}-t_{i}\right)\right] \\
& =E\left[\tilde{a}_{t_{i}} \operatorname{sign}_{\underline{\sigma}, \bar{\sigma}}\left(\tilde{a}_{t_{i}}\right)^{2}\left(t_{i+1}-t_{i}\right)-2 G\left(\tilde{a}_{t_{i}}\right)\left(t_{i+1}-t_{i}\right)\right]=0 .
\end{aligned}
$$

So

$$
E_{P_{\tilde{h}}}\left[-\left(K_{t}-K_{s}\right)\right] \leq 2 \mathbb{E}\left[\sup _{r \in[0,1]}\left|K_{r}-K_{r}(\zeta)\right|\right]<\varepsilon
$$

Lemma 3.5 Let $\mathcal{P} \subset \mathcal{M}_{1}\left(\Omega_{T}\right)$ be a weakly compact set that represents $\mathbb{E}$ :

$$
\mathbb{E}[\xi]=\sup _{Q \in \mathcal{P}} E_{Q}[\xi] \text { for all } \xi \in L_{i p}\left(\Omega_{T}\right) .
$$

Then, for $\xi \in L_{G}^{1}\left(\Omega_{T}\right),\left(E_{Q}[\xi]\right)_{Q \in \mathcal{P}}$ is continuous with respect to the weak convergence topology on $\mathcal{M}_{1}\left(\Omega_{T}\right)$. 
Proof. For $\xi \in L_{i p}\left(\Omega_{T}\right),\left(E_{Q}[\xi]\right)_{Q \in \mathcal{P}}$ is obviously continuous. By the definition of the space $L_{G}^{1}\left(\Omega_{T}\right)$, for $\xi \in L_{G}^{1}\left(\Omega_{T}\right),\left(E_{Q}[\xi]\right)_{Q \in \mathcal{P}}$ can be considered as the uniform limit of a sequence of continuous functions $\left(E_{Q}\left[\xi^{n}\right]\right)_{Q \in \mathcal{P}}$ with $\xi^{n} \in L_{i p}\left(\Omega_{T}\right)$ and $\mathbb{E}\left[\left|\xi^{n}-\xi\right|\right] \rightarrow 0$. So we get the desired result.

Theorem 3.6 Let $K_{t}=\int_{0}^{t} \eta_{s} d s$ for some $\eta \in M_{G}^{1}(0, T)$. If $K$ is a non-increasing $G$-martingale, we have $K \equiv 0$.

Proof. Without loss of generality, we consider the case $T=1$. Assume $\mathbb{E}\left[-K_{1}\right]>0$. Then, by Lemma 3.5, there exists $\varepsilon>0$ such that $\mathbb{E}_{G_{\varepsilon}}\left[-K_{1}\right]>0$. So, by Theorem 2.3 and Lemma 3.1 , we can find a process $h$ of form (3.1) with $\underline{\sigma}^{2}+\varepsilon \leq\left|h_{s}\right|^{2} \leq \bar{\sigma}^{2}-\varepsilon$ such that $\delta:=E_{P_{h}}\left[-K_{1}\right]>0$. Set $\alpha=\frac{\varepsilon}{\bar{\sigma}^{2}-\underline{\sigma}^{2}}$. For $k \geq 1$, set

$$
\left.\delta_{k, \alpha}(s)=\sum_{i=0}^{k-1}\left(1_{] \frac{i}{k}, \frac{i+\alpha}{k}\right]}(s)-1_{]} \frac{i+\alpha}{k}, \frac{i+1}{k}\right](s)\right) .
$$

Step 1. For any $n \geq 1$, we can find a $2^{n} m$-perturbation $h^{n}$ of $h$ with $\underline{\sigma} \leq\left|h_{s}^{n}\right| \leq \bar{\sigma}$ such that

$$
E_{P_{h^{n}}}\left[\int_{0}^{1} \delta_{2^{n} m, \alpha}^{+}(s) \eta_{s} d s\right]>-\frac{\delta}{2} \alpha .
$$

First, let us define $h_{s}^{n}$ for $s \in\left[0, \frac{1}{m}\right]$.

Set $\xi_{0}^{n}=\xi_{0}$.

By Lemma 3.4, there exists an $\mathbb{F}$-adapted measurable process $h^{n, 1,1}$ with $\underline{\sigma} \leq\left|h^{n, 1,1}\right| \leq \bar{\sigma}$ such that $E_{P_{h^{n, 1,1}}}\left[-\left(K_{\frac{\alpha}{2^{n} m}}-K_{0}\right)\right]<\frac{\delta}{2^{n+1} m} \alpha$.

Since $\sigma^{2}+\varepsilon \leq\left|\xi_{0}^{n}\right|^{2} \leq \bar{\sigma}^{2}-\varepsilon$, we have $\left.|| h_{s}^{n, 1,1}\right|^{2}-\left|\xi_{0}^{n}\right|^{2} \mid \leq \bar{\sigma}^{2}-\underline{\sigma}^{2}-\varepsilon$, by which we get $\left.\frac{\alpha}{1-\alpha}|| h_{s}^{n, 1,1}\right|^{2}-\left|\xi_{0}^{n}\right|^{2} \mid \leq \varepsilon$, and consequently

$$
\left.\frac{2^{n} m}{1-\alpha} \int_{0}^{\frac{\alpha}{2^{n} m}}|| h_{s}^{n, 1,1}\right|^{2}-\left|\xi_{0}^{n}\right|^{2} \mid d s \leq \varepsilon .
$$

So, noting $\underline{\sigma}^{2}+\varepsilon \leq\left|\xi_{0}^{n}\right|^{2} \leq \bar{\sigma}^{2}-\varepsilon$ again, we get

$$
\left|\xi_{0}^{n}\right|^{2}+\frac{2^{n} m}{1-\alpha} \int_{0}^{\frac{\alpha}{2^{n} m}}\left|\xi_{0}^{n}\right|^{2}-\left|h_{s}^{n, 1,1}\right|^{2} d s=\frac{1}{1-\alpha}\left(\left|\xi_{0}^{n}\right|^{2}-2^{n} m \int_{0}^{\frac{\alpha}{2^{n} m}}\left|h_{s}^{n, 1,1}\right|^{2} d s\right) \in\left[\underline{\sigma}^{2}, \bar{\sigma}^{2}\right] .
$$

Set

$$
h_{s}^{n}= \begin{cases}h_{s}^{n, 1,1} & \text { for } \left.s \in] 0, \frac{\alpha}{2^{n} m}\right] \\ \sqrt{\frac{1}{1-\alpha}\left(\left|\xi_{0}^{n}\right|^{2}-2^{n} m \int_{0}^{\frac{\alpha}{2^{n} m}}\left|h_{s}^{n, 1,1}\right|^{2} d s\right)} & \text { for } \left.s \in] \frac{\alpha}{2^{n} m}, \frac{1}{2^{n} m}\right] .\end{cases}
$$

It is easy to check that $\int_{0}^{\frac{1}{2^{n} m}}\left|h_{s}^{n}\right|^{2} d s=\frac{1}{2^{n} m}\left|\xi_{0}^{n}\right|^{2}$.

Assume that we have defined $h_{s}^{n}$ for all $s \in\left[0, \frac{j}{2^{n} m}\right], 0 \leq j \leq 2^{n}-1$. Then let us define $h_{s}^{n}$ for $\left.s \in] \frac{j}{2^{n} m}, \frac{j+1}{2^{n} m}\right]$.

By Lemma [3.4, there exists an $\mathbb{F}$-adapted process $h^{n, 1, j+1}$ with $\underline{\sigma} \leq\left|h^{n, 1, j+1}\right| \leq \bar{\sigma}$ and $h_{r}^{n, 1, j+1} 1_{\left[0, \frac{j}{2^{n} m}\right]}(r)=h_{r}^{n} 1_{\left[0, \frac{j}{2^{n} m}\right]}(r)$ such that $E_{P_{h^{n, 1, j+1}}}\left[-\left(K_{\frac{j+\alpha}{2^{n} m}}-K_{\frac{j}{2^{n} m}}\right)\right]<\frac{\delta}{2^{n+1} m} \alpha$.

Set

$$
h_{s}^{n}= \begin{cases}h_{s}^{n, 1, j+1} & \text { for } \left.s \in] \frac{j}{2^{n} m}, \frac{j+\alpha}{2^{n} m}\right] \\ \sqrt{\frac{1}{1-\alpha}\left(\left|\xi_{0}^{n}\right|^{2}-2^{n} m \int_{0}^{\frac{\alpha}{2^{n} m}}\left|h_{s}^{n, 1, j+1}\right|{ }^{2} d s\right)} & \text { for } \left.s \in] \frac{j+\alpha}{2^{n} m}, \frac{j+1}{2^{n} m}\right]\end{cases}
$$

It is easily seen that $\int_{\frac{j}{2^{n} m}}^{\frac{j+1}{2^{n} m}}\left|h_{s}^{n}\right|^{2} d s=\frac{1}{2^{n} m}\left|\xi_{0}^{n}\right|^{2}$ and $\left|h_{s}^{n}\right|^{2} \in\left[\underline{\sigma}^{2}, \bar{\sigma}^{2}\right]$. 
Assume that we have defined $h_{s}^{n}$ for all $s \in\left[0, \frac{i}{m}\right], 0 \leq i \leq m-1$.

Set $\xi_{i}^{n}=\varphi_{i}\left(\int_{\frac{i-1}{m}}^{\frac{i}{m}} h_{s}^{n} d W_{s}, \cdots, \int_{0}^{\frac{1}{m}} h_{s}^{n} d W_{s}\right)$.

Then we can define the process $h_{s}^{n}$ for $\left.\left.s \in\right] \frac{i}{m}, \frac{i+1}{m}\right]$ by repeating the above arguments with $\xi_{0}^{n}$ replaced by $\xi_{i}^{n}$.

Clealy, the process $h_{s}^{n}$ defined in this way is a $2^{n} m$-perturbation of $h$. Besides, we have

$$
E_{P_{h^{n}}}\left[\int_{0}^{1} \delta_{2^{n} m, \alpha}^{+}(s) \eta_{s}\right]=\sum_{j=0}^{2^{n} m-1} E_{P_{h^{n}}}\left[K_{\frac{j+\alpha}{2^{n} m}}-K_{\frac{j}{2^{n} m}}\right]>-\frac{\delta}{2} \alpha .
$$

Step 2. $\lim _{n \rightarrow \infty} \mathbb{E}\left[\left|\int_{0}^{1} \delta_{2^{n} m, \alpha}^{-}(s) \eta_{s} d s-(1-\alpha) K_{1}\right|\right]=0$.

For $\zeta \in M^{0}(0,1)$, the conclusion is obvious. As a functional of $\zeta \in M_{G}^{1}(0,1)$,

$$
D_{\alpha}(\zeta):=\limsup _{n \rightarrow \infty} \mathbb{E}\left[\left|\int_{0}^{1} \delta_{2^{n} m, \alpha}^{-}(s) \zeta_{s} d s-(1-\alpha) \int_{0}^{1} \zeta_{s} d s\right|\right]
$$

is continuous: $\left|D_{\alpha}(\zeta)-D_{\alpha}(\varsigma)\right| \leq\|\zeta-\varsigma\|_{M_{G}^{1}}$, for $\zeta, \varsigma \in M_{G}^{1}(0,1)$, which implies the desired result.

Step 3. $\lim _{n \rightarrow \infty} E_{P_{h^{n}}}\left[\int_{0}^{1} \delta_{2^{n} m, \alpha}^{-}(s) \eta_{s} d s\right]=(1-\alpha) E_{P_{h}}\left[K_{1}\right]=-(1-\alpha) \delta$.

Actually,

$$
\begin{aligned}
& \left|E_{P_{h^{n}}}\left[\int_{0}^{1} \delta_{2^{n} m, \alpha}^{-}(s) \eta_{s} d s\right]-(1-\alpha) E_{P_{h}}\left[K_{1}\right]\right| \\
\leq & \left|E_{P_{h^{n}}}\left[\int_{0}^{1} \delta_{2^{n} m, \alpha}^{-}(s) \eta_{s} d s\right]-(1-\alpha) E_{P_{h^{n}}}\left[K_{1}\right]\right|+(1-\alpha)\left|E_{P_{h^{n}}}\left[K_{1}\right]-E_{P_{h}}\left[K_{1}\right]\right| \\
\leq & \left.\mathbb{E}\left[\mid \int_{0}^{1} \delta_{2^{n} m, \alpha}^{-}(s) \eta_{s} d s\right]-(1-\alpha) K_{1} \mid\right]+(1-\alpha)\left|E_{P_{h^{n}}}\left[K_{1}\right]-E_{P_{h}}\left[K_{1}\right]\right| .
\end{aligned}
$$

By Step 2 and Theorem 3.3. we get the desired result.

Step 4. $\lim _{n \rightarrow \infty} \mathbb{E}\left[\int_{0}^{1}\left(\delta_{2^{n} m, \alpha}^{+}(s)-\frac{\alpha}{1-\alpha} \delta_{2^{n} m, \alpha}^{-}(s)\right) \eta_{s} d s\right]=0$.

The proof follows immediately from Step 2. Actually, setting

$$
d_{\alpha}(\zeta):=\limsup _{n \rightarrow \infty} \mathbb{E}\left[\left|\int_{0}^{1}\left(\delta_{2^{n} m, \alpha}^{+}(s)-\frac{\alpha}{1-\alpha} \delta_{2^{n} m, \alpha}^{-}(s)\right) \zeta_{s} d s\right|\right], \quad \zeta \in M_{G}^{1}(0,1),
$$

it is easily seen that $(1-\alpha) d_{\alpha}(\zeta)=D_{\alpha}(\zeta)$.

Combing the above arguments, we get

$$
\begin{aligned}
0 & =\lim _{n \rightarrow \infty} \mathbb{E}\left[\int_{0}^{1}\left(\delta_{2^{n} m, \alpha}(s)^{+}-\frac{\alpha}{1-\alpha} \delta_{2^{n} m, \alpha}(s)^{-}\right) \eta_{s} d s\right] \\
& \geq \limsup _{n \rightarrow \infty} E_{P_{h^{n}}}\left[\int_{0}^{1}\left(\delta_{2^{n} m, \alpha}^{+}(s)-\frac{\alpha}{1-\alpha} \delta_{2^{n} m, \alpha}^{-}(s)\right) \eta_{s} d s\right] \\
& \geq \limsup _{n \rightarrow \infty} E_{P_{h^{n}}}\left[\int_{0}^{1} \delta_{2^{n} m, \alpha}^{+}(s) \eta_{s}\right]-\frac{\alpha}{1-\alpha} \lim _{n \rightarrow \infty} E_{P_{h^{n}}}\left[\int_{0}^{1} \delta_{2^{n} m, \alpha}^{-}(s) \eta_{s} d s\right] \\
& \geq-\frac{\delta}{2} \alpha+\frac{\alpha}{1-\alpha} \times(1-\alpha) \delta=\frac{\delta}{2} \alpha>0,
\end{aligned}
$$

which is a contradiction. The last inequality follows from Step 1 and Step 3.

Corollary 3.7 Let $K_{t}=\int_{0}^{t} \eta_{s} d\langle B\rangle_{s}$ for some $\eta \in M_{G}^{1}(0, T)$. If $K$ is a non-increasing $G$ martingale, we have $K \equiv 0$. 
Proof. Set $L_{t}=\int_{0}^{t} \eta_{s} d s$. Assume that $K$ is a non-increasing $G$-martingale. Then we have

$$
0 \geq \mathbb{E}_{s}\left[L_{t}-L_{s}\right] \geq \frac{1}{\underline{\sigma}^{2}} \mathbb{E}_{s}\left[K_{t}-K_{s}\right]=0 .
$$

So $L$ is a non-increasing $G$-martingale. By Theorem 3.6, we get $L \equiv 0$, and consequently, $K \equiv 0$.

As an application of Theorem 3.6, we shall prove the uniqueness of the decomposition for generalized $G$-Itô processes.

Definition 3.8 A process of the following form is called a generalized G-Itô process:

$$
u=u_{0}+\int_{0}^{t} \eta_{s} d s+\int_{0}^{t} \zeta_{s} d B_{s}+K_{t}
$$

where $\eta \in M_{G}^{1}(0, T), \zeta \in H_{G}^{1}(0, T)$ and $K$ is a non-increasing $G$-martingale.

Remark 3.9 A G-Itô process

$$
u=u_{0}+\int_{0}^{t} \tau_{s} d s+\int_{0}^{t} \zeta_{s} d B_{s}+\int_{0}^{t} \frac{1}{2} \gamma_{s} d\langle B\rangle_{s}, \tau, \gamma \in M_{G}^{1}(0, T), \zeta \in H_{G}^{1}(0, T),
$$

can be rewritten as

$$
u=u_{0}+\int_{0}^{t}\left(\tau_{s}+G\left(\gamma_{s}\right)\right) d s+\int_{0}^{t} \zeta_{s} d B_{s}+K_{t},
$$

where $K_{t}=\int_{0}^{t} \frac{1}{2} \gamma_{s} d\langle B\rangle_{s}-\int_{0}^{t} G\left(\gamma_{s}\right) d s$, which, as is known, is a non-increasing G-martingale. So a $G$-Itô process is a generalized $G$-Itô process.

By Corollary 3.5 in Song (2012) we conclude that the decomposition for G-Itô processes is unique. The next result shows the uniqueness of the decomposition for generalized $G$-Itô processes.

Theorem 3.10 Assume $\int_{0}^{t} \zeta_{s} d B_{s}+\int_{0}^{t} \eta_{s} d s+K_{t}=L_{t}$, where $\zeta \in H_{G}^{1}(0, T), \eta \in M_{G}^{1}(0, T)$, and $K_{t}, L_{t}$ are non-increasing $G$-martingales. Then we have $\int_{0}^{t} \zeta_{s} d B_{s} \equiv 0, \int_{0}^{t} \eta_{s} d s \equiv 0$ and $K_{t}=L_{t}$.

Proof. By the uniqueness for the decomposition for (classical) continuous semimartingales, we get $\int_{0}^{t} \zeta_{s} d B_{s} \equiv 0$. Assume $\int_{0}^{t} \eta_{s} d s+K_{t}=L_{t}$. Since $L_{t}$ is non-increasing, $\tilde{L}_{t}:=\int_{0}^{t} \eta_{s}^{+} d s+K_{t}$ is also non-increasing, which implies that $-\int_{s}^{t} \eta_{r}^{+} d r \geq K_{t}-K_{s}$ for any $s<t$. Noting that $0 \geq \mathbb{E}_{s}\left[-\int_{s}^{t} \eta_{r}^{+} d r\right] \geq \mathbb{E}_{s}\left[K_{t}-K_{s}\right]=0$ since $K$ is a $G$-martingale, we conclude that $-\int_{0}^{t} \eta_{s}^{+} d s$ is also a $G$-martingale, which implies, by Theorem 3.6, that $\int_{0}^{t} \eta_{s}^{+} d s=0$. By the same arguments, we have $\int_{0}^{t} \eta_{s}^{-} d s=0$.

Corollary 3.11 Assume that $K_{t}:=\int_{0}^{t} \eta_{s} d\langle B\rangle_{s}-\int_{0}^{t} \zeta_{s} d s, \eta, \zeta \in M_{G}^{1}(0, T)$, is a non-increasing $G$-martingale. Then we have $\zeta \equiv 2 G(\eta)$.

Proof. Since $L_{t}:=\int_{0}^{t} \eta_{s} d\langle B\rangle_{s}-\int_{0}^{t} 2 G\left(\eta_{s}\right) d s=K_{t}+\int_{0}^{t} 2 G\left(\eta_{s}\right)-\zeta_{s} d s$ is a non-increasing $G$-martingale, by Theorem 3.10 , we get $\zeta \equiv 2 G(\eta)$.

Remark 3.12 Theorem 3.10 turns out to be a very strong conclusion. Many important results in the context of $G$-expectation theory can be considered as its immediate corollaries.

1) Theorem 3.6 in [12]: A process $A_{t}=\int_{0}^{t} \eta_{s} d\langle B\rangle_{s}, \eta \in M_{G}^{p}(0, T)$ for some $p>1$, has stationary and independent increments if and only if $A_{t}=c\langle B\rangle_{t}$ for some constant $c \in \mathbb{R}$. 
Proof. We only prove the "only if" part.

Assume that $A$ is a process with stationary and independent increments. Then there exists a constant $\lambda \in \mathbb{R}$ such that $\mathbb{E}\left[A_{t}\right]=\lambda t$ and $L_{t}:=A_{t}-\lambda t$ is a non-increasing $G$-martingale. So we conclude by Corollary 3.11 that $\lambda=2 G\left(\eta_{s}\right)$, which implies the desired conclusion.

2) Corollary 3.5 in [11]: If $\int_{0}^{t} \eta_{s} d\langle B\rangle_{s}=\int_{0}^{t} \zeta d s$ for some $\eta, \zeta \in M_{G}^{1}(0, T)$, we have $\eta=\zeta \equiv 0$. Proof. By the assumption, we have

$$
\int_{0}^{t} \eta_{s} d\langle B\rangle_{s}=: K_{t}+\int_{0}^{t} 2 G\left(\eta_{s}\right) d s=\int_{0}^{t} \zeta d s .
$$

By Theorem 3.10, we get $K_{t}=\int_{0}^{t} \eta_{s} d\langle B\rangle_{s}-\int_{0}^{t} 2 G\left(\eta_{s}\right) d s \equiv 0$. For any $\varepsilon \in\left[0, \frac{\bar{\sigma}^{2}-\underline{\sigma}^{2}}{2}\right]$ we have

$$
0=\mathbb{E}\left[-K_{T}\right] \geq \mathbb{E}_{G_{\varepsilon}}\left[-K_{T}\right] \geq \varepsilon \mathbb{E}_{G_{\varepsilon}}\left[\int_{0}^{T}\left|\eta_{s}\right| d s\right],
$$

which implies $\eta \equiv 0$, and consequently, $\zeta \equiv 0$.

\section{Application: characterization of $G$-Sobolev space $W_{\mathcal{A}_{G}}^{\frac{1}{2}, 1 ; p}(0, T)$}

Peng and Song (2015) introduced the notion of $G$-Sobolev spaces, in which they defined solutions to a certain type of path dependent PDEs.

Their definitions of $G$-Sobolev spaces started from the following spaces of smooth functions of paths.

Definition 4.1 A function $\xi: \Omega_{T} \rightarrow \mathbb{R}$ is called a cylinder function of paths on $[0, T]$ if it can be represented by

$$
\xi(\omega)=\varphi\left(\omega\left(t_{1}\right), \cdots, \omega\left(t_{n}\right)\right), \omega \in \Omega_{T},
$$

for some $0=t_{0}<\cdots<t_{n}=T$, where $\varphi:\left(\mathbb{R}^{d}\right)^{n} \rightarrow \mathbb{R}$ is a $C^{\infty}$-function with at most polynomial growth. We denote by $C^{\infty}\left(\Omega_{T}\right)$ the collection of all cylinder functions of paths on $[0, T]$.

Definition 4.2 A function $u(t, \omega):[0, T] \times \Omega_{T} \rightarrow \mathbb{R}$ is called a cylinder path process if there exists a time partition $0=t_{0}<\cdots<t_{n}=T$, such that for each $k=0,1, \cdots, n-1$ and $t \in\left(t_{k}, t_{k+1}\right]$,

$$
u(t, \omega)=u_{k}\left(t, \omega(t) ; \omega\left(t_{1}\right), \cdots, \omega\left(t_{k}\right)\right) .
$$

Here for each $k$, the function $u_{k}:\left[t_{k}, t_{k+1}\right] \times\left(\mathbb{R}^{d}\right)^{k+1} \rightarrow \mathbb{R}$ is a $C^{\infty}$-function with

$$
u_{k}\left(t_{k}, x ; x_{1}, \cdots, x_{k-1}, x\right)=u_{k-1}\left(t_{k}, x ; x_{1}, \cdots, x_{k-1}\right)
$$

such that, all derivatives of $u_{k}$ have at most polynomial growth. We denote by $\mathcal{C}^{\infty}(0, T)$ the collection of all cylinder path processes.

For a function $u \in \mathcal{C}^{\infty}(0, T)$, set, for $t \in\left[t_{k}, t_{k+1}\right]$,

$$
\begin{aligned}
& \mathcal{D}_{t} u(t, \omega):=\left.\partial_{t} u_{k}\left(t, x ; x_{1}, \cdots, x_{k}\right)\right|_{x=\omega(t), x_{1}=\omega\left(t_{1}\right), \cdots, x_{k}=\omega\left(t_{k}\right)}, \\
& \mathcal{D}_{x} u(t, \omega):=\left.\partial_{x} u_{k}\left(t, x ; x_{1}, \cdots, x_{k}\right)\right|_{x=\omega(t), x_{1}=\omega\left(t_{1}\right), \cdots, x_{k}=\omega\left(t_{k}\right)}, \\
& \mathcal{D}_{x}^{2} u(t, \omega):=\left.\partial_{x}^{2} u_{k}\left(t, x ; x_{1}, \cdots, x_{k}\right)\right|_{x=\omega(t), x_{1}=\omega\left(t_{1}\right), \cdots, x_{k}=\omega\left(t_{k}\right)}
\end{aligned}
$$

Set

$$
\mathcal{A}_{G} u(t, \omega):=\mathcal{D}_{t} u(t, \omega)+G\left(\mathcal{D}_{x}^{2} u(t, \omega)\right) .
$$




\subsection{G-Sobolev spaces $W_{G}^{1,2 ; p}(0, T)$ introduced in [7]}

Definition 4.3 1) For $u \in \mathcal{C}^{\infty}(0, T)$, we set

$$
\|u\|_{S_{G}^{p}}^{p}=\mathbb{E}\left[\sup _{s \in[0, T]}|u(s, \omega)|^{p}\right] .
$$

We denote by $S_{G}^{p}(0, T)$ the completion of $\mathcal{C}^{\infty}(0, T)$ w.r.t. the norm $\|\cdot\|_{S_{G}^{p}}$.

2) For $u \in \mathcal{C}^{\infty}(0, T)$, we set

$$
\|u\|_{W_{G}^{1,2 ; p}}^{p}=\mathbb{E}\left[\sup _{s \in[0, T]}\left|u_{s}\right|^{p}+\int_{0}^{T}\left(\left|\mathcal{D}_{s} u_{s}\right|^{p}+\left|\mathcal{D}_{x}^{2} u_{s}\right|^{p}\right) d s+\left(\int_{0}^{T}\left|\mathcal{D}_{x} u_{s}\right|^{2} d s\right)^{p / 2}\right] .
$$

Denote by $W_{G}^{1,2 ; p}(0, T)$ the completion of $\mathcal{C}^{\infty}(0, T)$ w.r.t. the norm $\|\cdot\|_{W_{G}^{1,2 ; p} \text {. }}$

Sometimes, we shall abuse notations by writing $u(t, \omega)$ as $u_{t}$ for simplicity.

By Corollary 3.5 in Song (2012) we conclude that the decomposition for $G$-Itô processes is unique: letting $\tau, \gamma \in M_{G}^{1}(0, T), \zeta \in H_{G}^{1}(0, T)$, then

$$
\int_{0}^{t} \tau_{s} d s+\int_{0}^{t} \zeta_{s} d B_{s}+\int_{0}^{t} \frac{1}{2} \gamma_{s} d\langle B\rangle_{s}=0
$$

implies that $\tau=\gamma \equiv 0$ and $\zeta \equiv 0$.

From this it is easily seen that the norm $\|\cdot\|_{W_{G}^{1,2 ; p}}$ is closable in the space $S_{G}^{p}(0, T)$ : Let

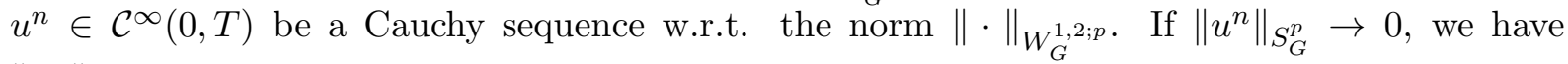
$\left\|u^{n}\right\|_{W_{G}^{1,2 ; p}} \rightarrow 0$.

Remark 4.4 The closability of the norm $\|\cdot\|_{W_{G}^{1,2 ; p}}$, which follows from the uniqueness of the decomposition for $G$-Itô processes, is the key point to extend the definition of the operators $\mathcal{D}_{t}$, $\mathcal{D}_{x} \mathcal{D}_{x}^{2}$ to the space $W_{G}^{1,2 ; p}(0, T)$. Precisely, unless the norm $\|\cdot\|_{W_{G}^{1,2 ; p}}$ is closable, a process $u \in S_{G}^{p}(0, T)$ may correspond to two different elements in $W_{G}^{1,2 ; p}(0, T)$, which can be represented as: $(u, \tau, \zeta, \gamma)$ and $(u, \tilde{\tau}, \tilde{\zeta}, \tilde{\gamma}), \tau, \tilde{\tau}, \gamma, \tilde{\gamma} \in M_{G}^{p}(0, T), \zeta, \tilde{\zeta} \in H_{G}^{p}(0, T)$. For this case, we could not well-define the derivatives for $u$.

So $W_{G}^{1,2 ; p}(0, T)$ can be considered as a subspace of $S_{G}^{p}(0, T)$, and the derivative operators $\mathcal{D}_{t}$, $\mathcal{D}_{x}^{2}$ (resp. $\left.\mathcal{D}_{x}\right)$, can all be extended as continuous linear operators from $W_{G}^{1,2 ; p}(0, T)$ to $M_{G}^{p}(0, T)$ (resp. to $H_{G}^{p}(0, T)$ ).

Theorem 4.5 (Theorem 4.5 in [7]) Assume $u \in S_{G}^{p}(0, T)$. Then the following two conditions are equivalent:

(i) $u \in W_{G}^{1,2 ; p}(0, T)$;

(ii) There exists $u_{0} \in \mathbb{R}, \zeta, w \in M_{G}^{p}(0, T)$ and $v \in H_{G}^{p}(0, T)$ such that

$$
u_{t}=u_{0}+\int_{0}^{t} \zeta_{s} d s+\int_{0}^{t} v_{s} d B_{s}+\frac{1}{2} \int_{0}^{t} w_{s} d\langle B\rangle_{s}
$$

Moreover, we have

$$
\mathcal{D}_{t} u_{t}=\zeta_{t}, \quad \mathcal{D}_{x} u_{t}=v_{t}, \quad \mathcal{D}_{x}^{2} u_{t}=w_{t} .
$$


In [7], the authors defined $W_{G}^{1,2 ; p}(0, T)$-solutions to path dependent PDEs and established one-one correspondence between backward SDEs under $G$-expectation and a certain type of path dependent PDEs.

Backward SDEs: to find $Y \in S_{G}^{p}(0, T), Z \in H_{G}^{p}(0, T), \eta \in M_{G}^{p}(0, T)$ such that

$$
Y_{t}=\xi+\int_{t}^{T} f\left(s, Y_{s}, Z_{s}, \eta_{s}\right) d s-\int_{t}^{T} Z_{s} d B_{s}-\left(\int_{t}^{T} \frac{1}{2} \eta_{s} d\langle B\rangle_{s}-\int_{t}^{T} G\left(\eta_{s}\right) d s\right),
$$

where $f:[0, T] \times \mathbb{R} \times \mathbb{R}^{d} \times \mathbb{S}(d) \mapsto \mathbb{R}$ is a given function and $\xi \in L_{G}^{p}\left(\Omega_{T}\right)$ is a given random variable.

Path Dependent PDEs: to find a path-dependent $u \in W_{G}^{1,2 ; p}(0, T)$ such that

$$
\begin{aligned}
\mathcal{D}_{t} u+G\left(\mathcal{D}_{x}^{2} u\right)+f\left(t, u, \mathcal{D}_{x} u, \mathcal{D}_{x}^{2} u\right) & =0, \quad t \in[0, T), \\
u_{T} & =\xi .
\end{aligned}
$$

We assume that $f\left(t, \omega, Y_{t}, Z_{t}, \eta_{t}\right) \in M_{G}^{p}(0, T)$ for any $(Y, Z, \eta) \in S_{G}^{p}(0, T) \times H_{G}^{p}(0, T) \times M_{G}^{p}(0, T)$.

Theorem 4.6 (Theorem 4.9 in [7]) Let $(Y, Z, \eta)$ be a solution to the backward SDE (4.5). Then we have $u_{t}:=Y_{t} \in W_{G}^{1,2 ; p}(0, T)$ with $\mathcal{D}_{x} u_{t}=Z_{t}$ and $\mathcal{D}_{x}^{2} u_{t}=\eta_{t}$.

Moreover, Given a $u \in W_{G}^{1,2 ; p}(0, T)$, the following conditions are equivalent:

(i) $\left(u, \mathcal{D}_{x} u, \mathcal{D}_{x}^{2} u\right)$ is a solution to the backward $S D E$ (4.5);

(ii) $u$ is a $W_{G}^{1,2 ; p}$-solution to the path dependent PDE (4.6).

\subsection{Characterization of $G$-Sobolev space $W_{\mathcal{A}_{G}}^{\frac{1}{2}, 1 ; p}(0, T)$}

Now we consider a special case of the path dependent PDE (4.6): $f$ is independent of $\mathcal{D}_{x}^{2} u$.

$$
\begin{aligned}
\mathcal{D}_{t} u+G\left(\mathcal{D}_{x}^{2} u\right)+f\left(t, u, \mathcal{D}_{x} u\right) & =0, \quad t \in[0, T), \\
u_{T} & =\xi .
\end{aligned}
$$

Let $u \in W_{G}^{1,2 ; p}(0, T)$ be a solution to the path dependent PDE (4.7). By Theorem 4.6, the processes

$$
Y_{t}:=u_{t}, Z_{t}:=\mathcal{D}_{x} u_{t}, K_{t}:=\frac{1}{2} \int_{0}^{t} \mathcal{D}_{x}^{2} u_{s} d\langle B\rangle_{s}-\int_{0}^{t} G\left(\mathcal{D}_{x}^{2} u_{s}\right) d s
$$

satisfy the following backward SDE:

$$
Y_{t}=\xi+\int_{t}^{T} f\left(s, Y_{s}, Z_{s}\right) d s-\int_{t}^{T} Z_{s} d B_{s}-\left(K_{T}-K_{t}\right),
$$

which is noting but the backward SDEs driven by $G$-Brownian motion ( $G$-BSDE) studied in [2].

On the contrary, letting $(Y, Z, K)$ be a solution of backward SDE (4.8) considered in [2], notice that, although we have many interesting examples, but it is still a very interesting and challenging problem to give reasonable conditions on $\xi$ and $f$ such that $Y$ lies in the Sobolev space $W_{G}^{1,2 ; p}(0, T)$. Even so, we still think $u=Y$ is a reasonable candidate of the solution to Equ. (4.7).

In [7], the authors formulated $u=Y$ as the unique solution to Equ. (4.7) in a first order Sobolev space $W_{\mathcal{A}_{G}}^{\frac{1}{2}, 1 ; p}(0, T)$. In this section, we shall refine the definition of the $G$-Sobolev space $W_{\mathcal{A}_{G}}^{\frac{1}{2}, 1 ; p}(0, T)$. The main idea is, just like the liner case, to integrate $\mathcal{A}_{G} u=\mathcal{D}_{t} u+G\left(\mathcal{D}_{x}^{2} u\right)$ as one operator, which reduces the regularity requirement for the solutions. To well define the derivative $\mathcal{A}_{G} u$ for $u \in W_{\mathcal{A}_{G}}^{\frac{1}{2}, 1 ; p}(0, T)$, the uniqueness of the decomposition for generalized $G$-Itô processes plays a crucial role. 


\subsubsection{Definition of the $G$-Sobolev space $W_{\mathcal{A}_{G}}^{\frac{1}{2}, 1 ; p}(0, T)$}

For $u, v \in \mathcal{C}^{\infty}(0, T)$, set

$$
d_{G, p}^{p}(u, v)=\mathbb{E}\left[\sup _{s \in[0, T]}\left|u_{s}-v_{s}\right|^{p}+\left(\int_{0}^{T}\left|\mathcal{D}_{x}\left(u_{s}-v_{s}\right)\right|^{2} d s\right)^{\frac{p}{2}}+\int_{0}^{T}\left|\mathcal{A}_{G} u_{s}-\mathcal{A}_{G} v_{s}\right|^{p} d s\right] .
$$

By the uniqueness of the decomposition for generalized $G$-Itô processes we obtain the closability of the metric $d_{G, p}$. As is stated in Remark 4.4, closability of the metric $d_{G, p}$ is the key point to well define the operators $\mathcal{A}_{G}, \mathcal{D}_{x}$.

Proposition 4.7 The metric $d_{G, p}$ is closable in the space $S_{G}^{p}(0, T)$ : Let $u^{n}, v^{n} \in \mathcal{C}^{\infty}(0, T)$ be two Cauchy sequences w.r.t. the metric $d_{G, p}$. If $\left\|u^{n}-v^{n}\right\|_{S_{G}^{p}} \rightarrow 0$, we have $d_{G, p}\left(u^{n}, v^{n}\right) \rightarrow 0$.

Proof. For $u^{n} \in \mathcal{C}^{\infty}(0, T)$, by Itô's formula, we have

$$
\begin{aligned}
u^{n}(t, \omega) & =u^{n}(0, \omega)+\int_{0}^{t} \mathcal{D}_{s} u^{n}(s, \omega) d s+\int_{0}^{t} \mathcal{D}_{x} u^{n}(s, \omega) d B_{s}+\frac{1}{2} \int_{0}^{t} \mathcal{D}_{x}^{2} u^{n}(s, \omega) d\langle B\rangle_{s} \\
& =u^{n}(0, \omega)+\int_{0}^{t} \mathcal{A}_{G} u^{n}(s, \omega) d s+\int_{0}^{t} \mathcal{D}_{x} u^{n}(s, \omega) d B_{s}+K_{t}^{n},
\end{aligned}
$$

where $K_{t}^{n}:=\frac{1}{2} \int_{0}^{t} \mathcal{D}_{x}^{2} u^{n}(s, \omega) d\langle B\rangle_{s}-\int_{0}^{t} G\left(\mathcal{D}_{x}^{2} u^{n}(s, \omega)\right) d s$ is a non-increasing $G$-martingale. If $\left(u^{n}\right)_{n}$ is a Cauchy sequence w.r.t. the metric $d_{G, p}$, there will be processes $u \in S_{G}^{p}(0, T), \eta \in$ $M_{G}^{p}(0, T), \zeta \in H_{G}^{p}(0, T)$ such that

$$
\left\|u^{n}-u\right\|_{S_{G}^{p}}+\left\|\mathcal{A}_{G} u^{n}-\eta\right\|_{M_{G}^{p}}+\left\|\mathcal{D}_{x} u^{n}-\zeta\right\|_{H_{G}^{p}} \rightarrow 0 .
$$

Set $K_{t}=u_{t}-u_{0}-\int_{0}^{t} \eta_{s} d s-\int_{0}^{t} \zeta_{s} d B_{s}$. It is easily seen that $\left\|K^{n}-K\right\|_{S_{G}^{p}} \rightarrow 0$. So $K$ is a nonincreasing $G$-martingale and $u_{t}=u_{0}+\int_{0}^{t} \eta_{s} d s+\int_{0}^{t} \zeta_{s} d B_{s}+K_{t}$ is a generalized $G$-Itô process. Assuming $\left(v_{n}\right)_{n}$ is a Cauchy sequence w.r.t. the metric $d_{G, p}$, similarly, there exists a generalized $G$-Itô process $\tilde{u}_{t}=\tilde{u}_{0}+\int_{0}^{t} \tilde{\eta}_{s} d s+\int_{0}^{t} \tilde{\zeta}_{s} d B_{s}+\tilde{K}_{t}$ such that

$$
\left\|v^{n}-\tilde{u}\right\|_{S_{G}^{p}}+\left\|\mathcal{A}_{G} v^{n}-\tilde{\eta}\right\|_{M_{G}^{p}}+\left\|\mathcal{D}_{x} v^{n}-\tilde{\zeta}\right\|_{H_{G}^{p}} \rightarrow 0 .
$$

If $\left\|u^{n}-v^{n}\right\|_{S_{G}^{p}} \rightarrow 0$, we get $u=\tilde{u}$. By the uniqueness of the decomposition for generalized $G$-Itô processes, we get $\eta=\tilde{\eta}$ and $\zeta=\tilde{\zeta}$, which implies $d_{G, p}\left(u^{n}, v^{n}\right) \rightarrow 0$.

Denote by $W_{\mathcal{A}_{G}}^{\frac{1}{2}, 1 ; p}(0, T)$ the closure of $\mathcal{C}^{\infty}(0, T)$ w.r.t. the metric $d_{G, p}$ in $S_{G}^{p}(0, T)$. Now the operators $\mathcal{A}_{G}, \mathcal{D}_{x}$ can be continuously extended to the space $W_{\mathcal{A}_{G}}^{\frac{1}{2}, 1 ; p}(0, T)$.

Proposition 4.8 Assume $u \in S_{G}^{p}(0, T)$. Then the following two conditions are equivalent:

(i) $u \in W_{\mathcal{A}_{G}}^{\frac{1}{2}, 1 ; p}(0, T)$;

(ii) There exists $\eta \in M_{G}^{p}(0, T)$ and $\zeta \in H_{G}^{p}(0, T)$ such that

$$
u(t, \omega)-\int_{0}^{t} \eta(s, \omega) d s-\int_{0}^{t} \zeta(s, \omega) d B_{s}
$$

is a non-increasing $G$-martingale, namely, $u$ is a generalized $G$-Itô process.

Moreover, we have $\mathcal{A}_{G} u=\eta$ and $\mathcal{D}_{x} u=\zeta$. 
Proof. (i) $\Longrightarrow$ (ii) is obvious.

(ii) $\Longrightarrow$ (i). Let $u$ be a generalized $G$-Itô process. By Theorem 5.4 in [10], it suffices to prove the claim for $u$ of the following form:

$$
u_{t}=u_{0}+\int_{0}^{t} \eta_{s} d s+\int_{0}^{t} \zeta_{s} d B_{s}+\frac{1}{2} \int_{0}^{t} w_{s} d\langle B\rangle_{s}-\int_{0}^{t} G\left(w_{s}\right) d s
$$

where $\eta, \zeta, w$ are smooth step processes, namely, $\eta, \zeta, w \in M^{0}(0, T)$ and $\eta_{t}, \zeta_{t}, w_{t} \in C^{\infty}\left(\Omega_{t}\right)$ (Def. 4.1). Set $t_{k}^{n}=\frac{k T}{2^{n}}$ and $Q_{t}^{n}:=\sum_{k=0}^{2^{n}-1}\left(B_{t_{k+1}^{n} \wedge t}-B_{t_{k}^{n} \wedge t}\right)^{2}=\int_{0}^{t} \lambda_{s}^{n} d B_{s}+\langle B\rangle_{t}$, where $\lambda_{t}^{n}=\sum_{k=0}^{2^{n}-1} 2\left(B_{t}-B_{t_{k}}\right) 1_{\left.t_{k}, t_{k+1}\right]}(t)$. Choose a sequence of smooth step processes $\alpha_{s}^{n}$ such that $\mathbb{E}\left[\int_{0}^{T}\left|\alpha_{s}^{n}-G\left(w_{s}\right)\right|^{p} d s\right] \rightarrow 0$. Set

$$
\begin{aligned}
u_{t}^{n} & :=u_{0}+\int_{0}^{t} \eta_{s} d s+\int_{0}^{t} \zeta_{s} d B_{s}+\frac{1}{2} \int_{0}^{t} w_{s} d Q_{s}^{n}-\int_{0}^{t} \alpha_{s}^{n} d s \\
& =u_{0}+\int_{0}^{t}\left(\eta_{s}-\alpha_{s}^{n}\right) d s+\int_{0}^{t}\left(\zeta_{s}+\frac{1}{2} w_{s} \lambda_{s}^{n}\right) d B_{s}+\int_{0}^{t} \frac{1}{2} w_{s} d\langle B\rangle_{s} .
\end{aligned}
$$

It is easily seen, by (4.9), that $u^{n}$ belongs to $\mathcal{C}^{\infty}(0, T)$. By the uniqueness of the decomposition for $G$-Itô processes and (4.10) we know that

$$
\mathcal{D}_{t} u_{t}^{n}=\eta_{t}-\alpha_{t}^{n}, \mathcal{D}_{x} u_{t}^{n}=\zeta_{t}+\frac{1}{2} w_{t} \lambda_{t}^{n}, \mathcal{D}_{x}^{2} u_{t}^{n}=w_{t}
$$

So $\mathcal{A}_{G} u_{t}^{n}=\eta_{t}-\alpha_{t}^{n}+G\left(w_{t}\right)$. It is easy to show that $\mathcal{A}_{G} u^{n} \stackrel{M_{G}^{p}}{\longrightarrow} \eta, D_{x} u^{n} \stackrel{H_{G}^{p}}{\longrightarrow} \zeta, u^{n} \stackrel{S_{G}^{p}}{\longrightarrow} u$. So $u \in W_{\mathcal{A}_{G}}^{\frac{1}{2}, 1 ; p}(0, T)$ with $\mathcal{A}_{G} u=\eta$ and $\mathcal{D}_{x} u=\zeta$.

\subsubsection{Fully nonlinear path dependent PDEs}

Let us define the $W_{\mathcal{A}_{G}}^{\frac{1}{2}, 1, p}$-solution to the path dependent PDE (4.7) $:$ to find $u \in W_{\mathcal{A}_{G}}^{\frac{1}{2}, 1 ; p}(0, T)$ such that

$$
\begin{aligned}
\mathcal{A}_{G} u+f\left(t, u, \mathcal{D}_{x} u\right) & =0, \quad t \in[0, T), \\
u_{T} & =\xi .
\end{aligned}
$$

Now we can interpret backward SDEs driven by $G$-Brownian motion as "path dependent" PDEs.

We assume that $f\left(t, \omega, Y_{t}, Z_{t}\right) \in M_{G}^{p}(0, T)$ for any $(Y, Z) \in S_{G}^{p}(0, T) \times H_{G}^{p}(0, T)$.

Theorem 4.9 Let $(Y, Z)$ be a solution to the backward SDE 4.8) (see Def. 5.1 and Rem. 5.3). Then we have $u_{t}:=Y_{t} \in W_{\mathcal{A}_{G}}^{\frac{1}{2}, 1 ; p}(0, T)$ with $\mathcal{D}_{x} u_{t}=Z_{t}$.

Moreover, Given a $u \in W_{\mathcal{A}_{G}}^{\frac{1}{2}, 1 ; p}(0, T)$, the following conditions are equivalent:

(i) $\left(u, \mathcal{D}_{x} u\right)$ is a solution to the backward $S D E$ [4.8);

(ii) $u$ is a $W_{\mathcal{A}_{G}}^{\frac{1}{2}, 1 ; p}(0, T)$-solution to the path dependent PDE 4.11).

Proof. The proof follows immediately from Proposition 4.8 and the definitions of the solutions to the backward SDE (4.8) and the path dependent PDE (4.11).

Assume that the function $g(t, \omega, y, z):[0, T] \times \Omega_{T} \times R \times R \rightarrow R$ satisfies the following assumptions: there exists some $\beta>1$ such that

(H1) for any $y, z, g(t, \omega, y, z) \in M_{G}^{\beta}(0, T)$;

(H2) $\left|g(t, \omega, y, z)-g\left(t, \omega, y^{\prime}, z^{\prime}\right)\right| \leq L\left(\left|y-y^{\prime}\right|+\left|z-z^{\prime}\right|\right)$ for some constant $L>0$. 
Corollary 4.10 Assume $\xi \in L_{G}^{\beta}\left(\Omega_{T}\right)$ and $g$ satisfies (H1) and (H2) for some $\beta>1$. Then, for each $p \in(1, \beta)$, the path dependent PDE 4.7) has a unique $W_{\mathcal{A}_{G}}^{\frac{1}{2}, 1 ; p}$-solution $u$.

In particular, the martingale $u(t, \omega):=\mathbb{E}_{t}[\xi](\omega)$ is the unique $W_{\mathcal{A}_{G}}^{\frac{1}{2}, 1 ; p}$-solution of the path dependent G-heat equation

$$
\mathcal{D}_{t} u+G\left(\mathcal{D}_{x}^{2} u\right)=0, \quad u_{T}=\xi .
$$

Proof. The uniqueness is straightforward from Theorem 4.9 and Theorem 5.2 .

We now prove the existence. By Theorem 5.2 we know that the backward SDE (4.8) has a solution $(Y, Z)$. By the assumption (H1) and (H2), we conclude $g\left(t, \omega, Y_{t}(\omega), Z_{t}(\omega)\right) \in M_{G}^{p}(0, T)$. So we get the existence result from Theorem 4.9.

By the $G$-martingale decomposition theorem, $u \in S_{G}^{p}(0, T)$ is a $G$-martingale if and only if $u$ is a solution of backward $\operatorname{SDE}(4.8)$ with $f=0$. So $u(t, \omega):=\mathbb{E}_{t}[\xi](\omega)$ is the unique $W_{\mathcal{A}_{G}}^{\frac{1}{2}, 1 ; p}$-solution of the path dependent $G$-heat equation.

\section{Appendix: Backward SDEs driven by $G$-Brownian motion}

In [2] the authors studied the backward stochastic differential equations driven by a $G$-Brownian motion $\left(B_{t}\right)_{t \geq 0}$ in the following form:

$$
Y_{t}=\xi+\int_{t}^{T} g\left(s, Y_{s}, Z_{s}\right) d s-\int_{t}^{T} Z_{s} d B_{s}-\left(K_{T}-K_{t}\right)
$$

where $K$ is a non-increasing $G$-martingale.

The main result in [2] is the existence and uniqueness of a solution $(Y, Z, K)$ for equation (5.1) in the $G$-framework under the following assumption: there exists some $\beta>1$ such that (H1) and (H2) are satisfied.

Definition 5.1 Let $\xi \in L_{G}^{\beta}\left(\Omega_{T}\right)$ and $g$ satisfy (H1) and (H2) for some $\beta>1$. A triplet of processes $(Y, Z, K)$ is called a solution of equation (5.1) if for some $1<\alpha \leq \beta$ the following properties hold:

(a) $Y \in S_{G}^{\alpha}(0, T), Z \in H_{G}^{\alpha}(0, T), K$ is a non-increasing $G$-martingale with $K_{0}=0$ and $K_{T} \in$ $L_{G}^{\alpha}\left(\Omega_{T}\right)$

(b) $Y_{t}=\xi+\int_{t}^{T} g\left(s, Y_{s}, Z_{s}\right) d s-\int_{t}^{T} Z_{s} d B_{s}-\left(K_{T}-K_{t}\right)$.

The main result in [2] is the following theorem:

Theorem 5.2 Assume that $\xi \in L_{G}^{\beta}\left(\Omega_{T}\right)$ and $f$ satisfies (H1) and (H2) for some $\beta>1$. Then equation (5.1) has a unique solution $(Y, Z, K)$. Moreover, for any $1<\alpha<\beta$ we have $Y \in$ $S_{G}^{\alpha}(0, T), Z \in H_{G}^{\alpha}(0, T)$ and $K_{T} \in L_{G}^{\alpha}\left(\Omega_{T}\right)$.

Remark 5.3 Equivalently, we say a pair of processes $(Y, Z)$ is a solution of equation (5.1) if for some $1<\alpha \leq \beta$ the following properties hold:

(a) $Y \in S_{G}^{\alpha}(0, T), Z \in H_{G}^{\alpha}(0, T)$;

(b) $Y_{T}=\xi$ and $K_{t}:=Y_{t}+\int_{0}^{t} g\left(s, Y_{s}, Z_{s}\right) d s-\int_{0}^{t} Z_{s} d B_{s}$ is a non-increasing $G$-martingale. 


\section{References}

[1] Denis, L., Hu, M. and Peng S.(2011) Function spaces and capacity related to a sublinear expectation: application to G-Brownian motion paths, Potential Anal., 34: 139-161.

[2] Hu, M., Ji, S., Peng, S. and Song, Y. (2014) Backward Stochastic Differential Equations Driven by G-Brownian Motion, Stochastic Processes and their Applications 124, 759-784.

[3] Peng, S. (2007a) G-Expectation, G-Brownian motion and related stochastic calculus of Itô's type, Stochastic Analysis and Applications, The Abel Symposium 2005, Abel Symposia, Edit. Benth et. al., 541-567, Springer-Verlag.

[4] Peng, S. (2007b) G-Brownian Motion and Dynamic Risk Measure under Volatility Uncertainty, arXiv:0711.2834v1 [math.PR].

[5] Peng, S. (2008) Multi-Dimensional G-Brownian Motion and Related Stochastic Calculus under G-Expectation, Stochastic Processes and their Applications, 118(12): 2223-2253.

[6] Peng, S. (2010) Nonlinear Expectations and Stochastic Calculus under Uncertainty, preprint, arXiv:1002.4546.

[7] Peng, S. and Song, Y. G-expectation weighted Sobolev spaces, backward SDE and path dependent PDE, J. Math. Soc. Japan Vol. 67, No. 4, pp. 17251757.

[8] Soner M, Touzi N, Zhang J.(2012) Well-posedness of Second Order Backward SDEs, Probability Theory and Related Fields, 153(1-2): 149-190.

[9] Song, Y.(2011) Some properties on G-evaluation and its applications to G-martingale decomposition, Science China Mathematics, 54(2): 287-300.

[10] Song, Y.(2011) Properties of hitting times for G-martingales and their applications, Stochastic Processes and their Applications, 121: 1770-1784.

[11] Song, Y.(2012) Uniqueness of the representation for G-martingales with finite variation, Electron. J. Probab. 17 no. 24 1-15.

[12] Song, Y.(2013) Characterizations of processes with stationary and independent increments under G-expectation, Annales de l'Institut Henri Poincare, Vol. 49, No. 1, 252-269. 